\section{Molecular Biology Becomes Diagnostic Tool in the Newborn Emergency Department: A Rare Case of Child Idiopathic Arterial Calcification}

\section{Abstract}

A Turkish infant of 96 days is accepted to the pediatric emergency room after being carried by their parents because of inconsolable crying, persistent cough, and difficulty in feeding during the previous day.

Although she was aware and nor signs nor symptoms was suggestive of an impending MOF, in a few minutes it takes over an asystolic cardiac arrest. It was found the exitus after 75 minutes of resuscitation.

The autopsy report indicates as pathological cause of death a rare form of childhood idiopathic calcific arterial disease caused by mutation of the gene encoding the same name EPP1 enzyme.

Keywords: Newborn; Idiopathic; Diagnostic tool

Received: November 09, 2015; Accepted: December 10, 2015; Published: December 20, 2015

\section{Clinical Case}

This case report aims to share with readers the experience of the medical team of the hospital A. Manzoni in Lecco, who has had to handle a sudden cardiac arrest in an infant of three months old, a context in which the clinical features remained unclear and blurred all the time of resuscitation, until the exploration autopsy and pathological investigation outlined in its components.

The baby, 96 days old, had been conducted in pediatric emergency from the parents, because during the night she had appeared agitated; parents also reported excesses of uncontrollable coughing and power failure during the previous 12 hours. The patient was hypothermic $\left(35.9^{\circ} \mathrm{C}\right.$ rectal temperature), with episodes of unconsolable crying; then she was borught to the attention of a pediatrician with a priority value equal to 4 (noncritical) $[1,2]$.

Physical examination shows fairly good general conditions, weight of 4, 820 grams (reportedly on the rise), drowsiness Glasgow Coma Scale=11 (be awakened by a painful stimulus), plaintive cry, photometer reflex present; no meningeal signs; Moro reflex present; pale-pink skin with signs of hypothermia over the extremities and perioral cyanosis; breath leaning; at auscultation chest the murmur was tightened, but without the presence of noise added; no organomegaly. Given the difficulties in finding a

\author{
Andrea Borromini", \\ Giorgio Rossi ${ }^{2}$, \\ Paola Maggi ${ }^{3}$, \\ Daniele Speciale', \\ Giampaolo Mirri ${ }^{4}$, \\ Anna Cogliardi ${ }^{3}$, \\ Claudia Addis ${ }^{3}$, \\ Emanuele Dainese ${ }^{2}$, \\ Emanuela Bonoldi ${ }^{2}$ and \\ Alessandro Marando ${ }^{2}$
}

1 Department of Emergency Medicine, Division of Anesthesiology and Intensive Care - H. Alessandro Manzoni, Lecco, Italy

2 Department of Laboratory Medicine, Division of Pathology - H. Alessandro Manzoni, Lecco, Italy

3 Department of Mother and Child, Division of Pediatrics - H. Alessandro Manzoni, Lecco, Italy

4 Department of Mother and Child, Division of Neonatology and Neonatal Intensive Care- H. Alessandro Manzoni, Lecco, Italy

\section{Corresponding Author:} Andrea Borromini

$\equiv$ andrea.borromini01@niversitadipavia.it

Department of Emergency Medicine, Division of Anesthesiology and Intensive Care - H. Alessandro Manzoni, Lecco, Italy.

Citation: Borromini A, Rossi G, Maggi P, et al. Molecular Biology Becomes Diagnostic Tool in the Newborn Emergency Department: A Rare Case of Child Idiopathic Arterial Calcification. J Pediatr Care. 2015, 1:1.

peripheral venous access, the anesthetist and the neonatologist were contacted to collaborate in assisting the infant $[3,4]$.

On their arrival the general conditions were already deteriorating. The breath had become rapidly tachi-dyspnoeic and therapy with oxygen mask with reservoir was started. The peripheral 
saturation of hemoglobin was not assessable as well as the noninvasive blood pressure. The absence of jugular turgor and hepatosplenomegaly were evaluated and fluids were administered through intraosseous tibial as anti-shock rescue; however, the non-invasive blood pressure remains invaluable.

Suddenly the patient, meanwhile cardiorespiratory monitored, undergoes ACC asystolic. She was immediately and successfully intubated by nasotracheal way; after a second way of intraosseous was taken, we begun resuscitation as guidelines. At the same time the internal jugular vein is cannulated through eco- driving, which is drawing blood for urgent blood tests and blood gas analysis: they showed the presence of severe metabolic acidosis and severe electrolyte disorder which was corrected immediately be infusion of sodium bicarbonate and calcium chloride, but we were unable to restore a level of metabolic homeostasis [5].

Also contacted the cardiologist, echocardiography is performed continuously showing the absence of ventricular mechanical activity. After 75 minutes of resuscitation, during which there has been no resumption of shockable rhythm, it finds death.

After three days it runs autopsy, requesting confirmation for alleged and suspected heart disease.

However, the report states the presence of a condition much more complex and articulated one of the cardiopathy.

In fact, althought there were no macroscopic suggestive signs, istologically, it sets out a framework of multi-organ failure from Infantile Idiopathic arterial calcification (IIAC) which affected the arteries of medium and large caliber of the major organs (Figures 1-5) such as the heart (Figure 5)-coronary branches-kidneys (Figure 3), liver, lungs (Figures 1 and 4) where, at this level, aspects of luminal stenosis, arterial thrombosis were observed, resulting in such extended areas of ischemic necrosis haemorrhagic interesting large part of the right lower lobe and smaller focal areas in the remaining districts lung bilaterally.

Rare cases [6] of calcific Areriopatia Infantile Idiopathic are reported in the literature as an expression of autosomal

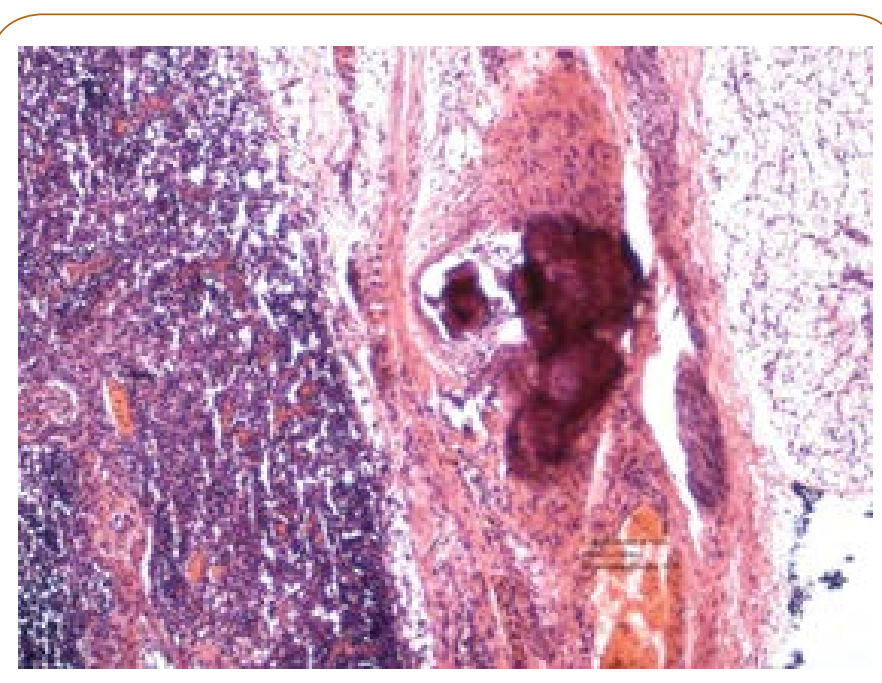

Figure 1 Lung arterial vessel calcification; hematoxylin-eosin.

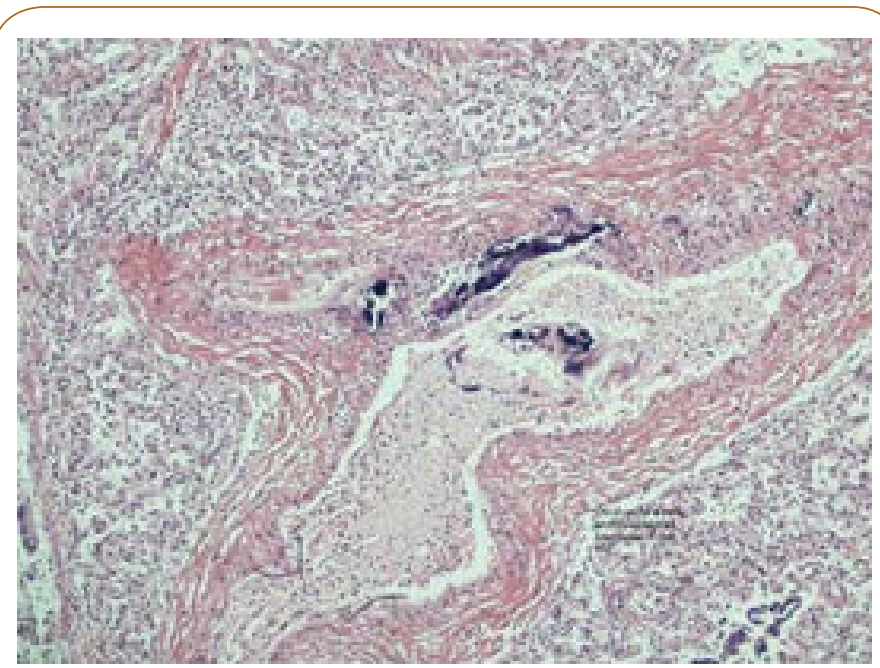

Figure 2 Thymic arterial vessel calcification; hematoxylineosin.

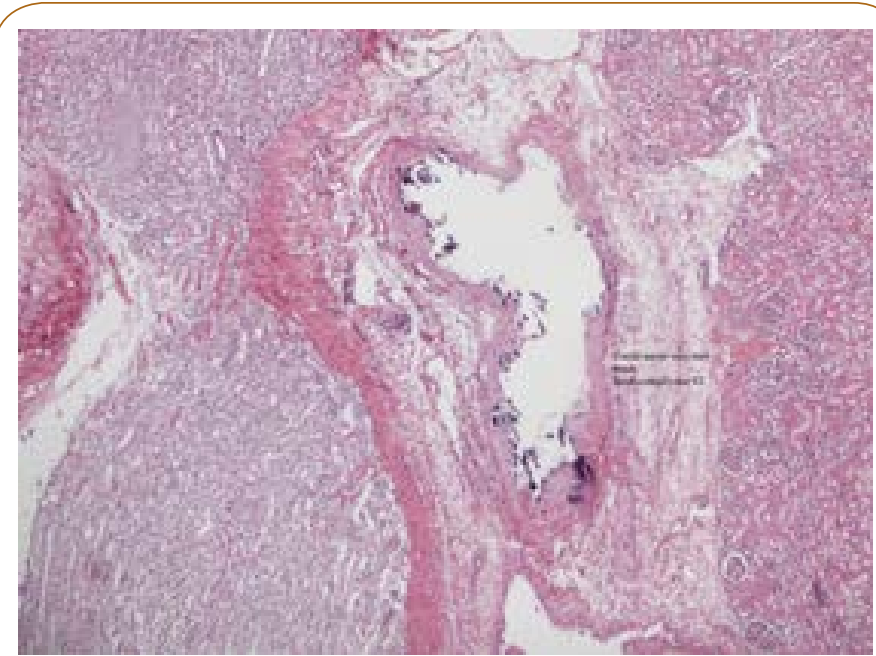

Figure 3 Kidney arterial vessel calcification; hematoxylineosin.

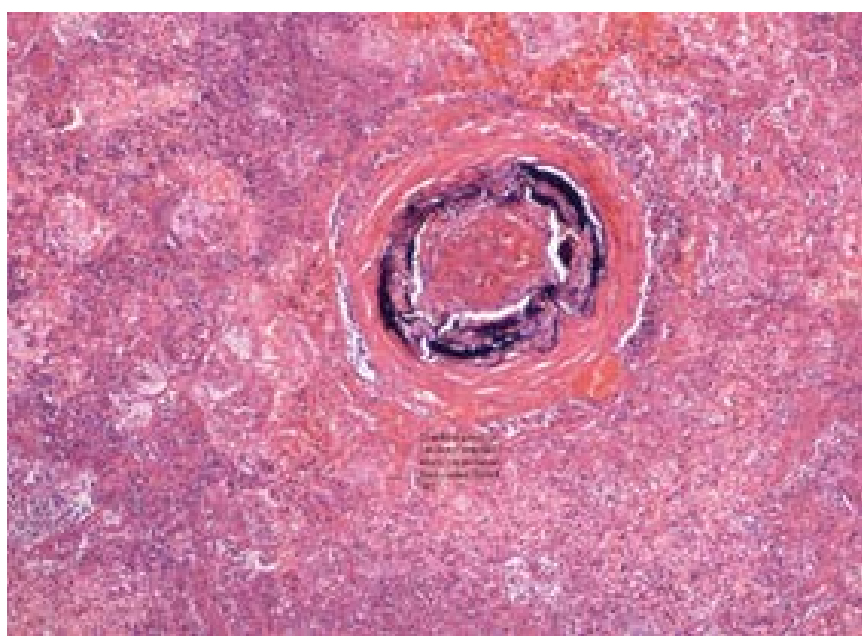

Figure 4 Calcific arterial thrombosis with acute lung parenchyma necrotic infarct; hematoxylin-eosin. 


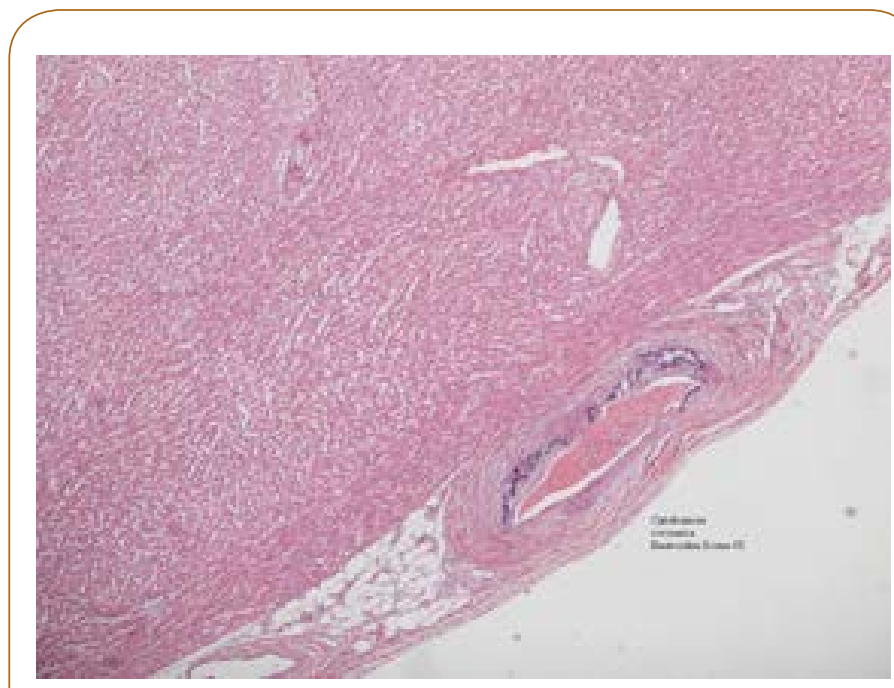

Figure 5 Coronaric calcification; hematoxylin-eosin.

recessive gene mutation condificante ENPP1 (Ectonucleotide pyrophosphatase/phosphodiesterase 1).

\section{Discussion}

The review showed that the pathology is corresponding to very limited circumstances described in the literature, [6] which presents histological and microscopic similar features: widespread calcification blood vessels that it causes extensive hemorrhagic-necrotic areas hesitate then within a framework of multi- organ failure irreversible.

Generalized arterial calcification of infancy (GACI) is an autosomal recessive disorder caused by mutations in the gene ENPP1, an enzyme that hydrolyzes ATP to AMP and inorganic pyrophosphate (PPI). Under physiological conditions, PPi serves as a powerful antimineralization factor and the reduced activity of ENPP1 brings to a pro - mineralizing environment and promotes mineralization in ectopic tissues.

Individuals affect die within the first year of life in many cases and there is currently no effective treatment for this disease; However, the clinical suspicion of the condition can be confirmed with imaging studies, arterial biopsy and blood investigations of molecular biology, in order to establish an early bisphosphonate therapy, which proved effective in reducing ectopic calcific deposits and extending life expectancy.

It's good medical practice, once the suspected diagnosis is confirmed, investigating genetic condition through appropriate specialist valuation, so as to inform the family. In addition, previous diagnostic confirmation may be a valuable guide in the management of future pregnancies in families with known presence or absence of the mutation, so that investigations and molecular prenatal ultrasound can be early performed.
Through the presentation of this case report, we want to bring out two fundamental aspects of medical practice: on the one hand the indispensable need to field the skills of several disciplines to achieve a common goal, which is the formulation of a diagnosis and a subsequent adequate treatment. This is even more evident in a case like the one described, in which the management of an individual patient required the presence of most medical specialists to cope with problems: pediatric, intensive care, cardiology. The ability to identify a team leader who would manage the overall event and orchestrate the various components of the medical team, has allowed us to optimize resources without neglecting any detail, then despite the negative outcome. Next to the common clinical practice, in this case pathologic medicine is inserted so impressive even often little assimilated to the most common medical disciplines, but of primary importance in order to reach a definitive diagnosis.

Another interesting point to ponder is that, increasingly in recent years, we are witnessing a transition and translation of medical practice, no longer limited to the process of clinical investigation and subsequent synthesis diagnosis and treatment, but to a more in-depth analysis that aims to shed light on the molecular and biochemical mechanism responsible for the phenotype that you are facing. The exploration is gradually becoming deeper and deeper, demanding in this sense a continuous training in the medical and scientific bases, to bio-molecular. There is therefore a care pathway that sees its development interlinking of more medical figures, starting with the management in the department of pediatrics, to get to close and will result in a more microscopic evaluation through the work of pathologists until the discovery of how the mutation of a gene encoding an enzyme is responsible for a rare childhood disease that leads to widespread arterial calcification, resulting, as is clear from the literature, in death in all cases.

\section{Take Home Messages of the Clinical Case}

- The clinical conditions of an infant, who is brought to the attention of the medical pediatric first aid, can rapidly deteriorate leading to situation increasingly difficult clinical management: you must always be alert and ready to face any ominous development.

- During the emergency in children seeking the cooperation of various specialists is very good in order to have all the tools at the right time.

- The pathology and molecular biology tools become indispensable in clinical practice where it is highly difficult to make a diagnosis through routine clinical practice.

- This case emphasizes the importance of taking into consideration the idiopathic infantile arterial calcification as a differential diagnosis in all cases of cardiopulmonary failure in the infants. 


\section{References}

1 Li Q (2014) Spontaneous asj-2J mutant mouse as a model for generalized arterial calcification of infancy: a large deletion/insertion mutation in the Enpp1 gene. PLoS One 5: 9(12).

2 Ferreira C (2014) Generalized Arterial Calcification of Infancy. GeneReviews ${ }^{\circledR}$. Seattle (WA): University of Washington, Seattle; 1993-2015.

3 Apschner A (2014) Zebrafish enpp1 mutants exhibit pathological mineralization, mimicking features of generalized arterial calcification of infancy (GACl) and pseudoxanthoma elasticum (PXE). Dis Model Mech.

4 Hofmann Bowman MA (2012) Genetic pathways of vascular calcification. Trends Cardiovasc Med 22(4).

5 Kalal IG (2012) Molecular diagnosis of generalized arterial calcification of infancy (GACl). J Cardiovasc Dis Res 3(2).

6 Neal A (2014) Idiopathic Infantile Arterial Calcification: a possible cause of refractory cardiopulmonary failure in infancy. Case Reports in Pathology. 